\title{
A Transformation Method for the Reconstruction of Functions from Nonuniformly Spaced Samples
}

\author{
JAMES J. CLARK, MATTHEW R. PALMER, AND PETER D. LAWRENCE, MEMBER, IEEE
}

\begin{abstract}
The reconstruction of functions from their samples at nonuniformly distributed locations is an important task for many applications. This paper presents a sampling theory which extends the uniform sampling theory of Whittaker $e t$ al. [11] to include nonuniform sample distributions. This extension is similar to the analysis of Papoulis [15], who considered reconstructions of functions that had been sampled at positions deviating slightly from a uniform sequence. Instead of treating the sample sequence as deviating from a uniform sequence, we show that a more general result can be obtained by treating the sample sequence as the result of applying a coordinate transformation to the uniform sequence. It is shown that the class of functions reconstructible in this manner generally include nonband-limited functions.

The two-dimensional uniform sampling theory of Petersen and Middleton [16] can be similarly extended as is shown in this paper.

A practical algorithm for performing reconstructions of two-dimensional functions from nonuniformly spaced samples is described, as well as examples illustrating the performance of the algorithm.
\end{abstract}

\section{INTRODUCTION}

$\mathrm{I}$ $\mathrm{N}$ many applications it is required that a function of one or Lmore variables be reconstructed from knowledge of its values at nonuniformly distributed sample locations. These applications are found in such diverse fields as machine vision [5] and [17], radio astronomy [9], and computed tomography [14]. Methods that have been used to perform such reconstructions include nearest neighbour and bilinear interpolation [14], surface functional minimization by relaxation [17] or gradient descent methods [5], and Gaussian smearing-resampling [9]. These methods, however, either do not result in a minimum possible reconstruction error or they require $a$ priori knowledge about the form of the function.

Other techniques, based on nonharmonic Fourier series, have been proposed [3], [8], [18] for reconstructing bandlimited one-dimensional functions that have been sampled at irregular intervals. These methods, however, are limited to sample sequences which have only minor deviations from a uniform sampling sequence. Such techniques are inapplicable to situations wherein the sample density varies significantly. In Section II of this paper we develop a one-dimensional sampling theory, which we extend to two dimensions in Section III, that allows one, under certain conditions, to reconstruct band-limited functions exactly from arbitrarily distributed samples. This theory is seen to be equivalent to the analysis of Papoulis [15], who showed how the standard

Manuscript received May 15, 1984; revised March 5, 1985. This work was supported by the B.C. Science Council under Grant (52(RCG-8)) and by B.C. Science Council G.R.E.A.T. scholarships.

The authors are with the Department of Electrical Engineering, University of British Columbia, Vancouver, B.C., Canada V6T-1W5. uniform sampling theory of Whittaker et al. [11] could be extended to sample sequences that were slight deviations from a uniform sample sequence. In this paper we show how a more general result can be obtained by treating a nonuniform sample sequence as resulting from a coordinate transformation of a uniform sample sequence instead of being merely deviated from the uniform sequence.

Section IV details how these coordinate transformations can be determined in the two-dimensional case. Section $\mathrm{V}$ describes a heuristic algorithm, based on the theory developed in Section III, for performing two-dimensional function reconstruction from nonuniformly distributed samples.

Examples of one- and two-dimensional function reconstruction can be found in Section VI.

Proofs of theorems stated in this paper can be found in the Appendix.

\section{One-Dimensional Sampling Theory}

We will first derive a nonuniform sampling theorem for the one-dimensional case and then extend this result to the twodimensional case in Section III.

The starting point for our derivation is the classical Whittaker-Kotel'nikov-Shannon (WKS) sampling theorem (Theorem 1) (see the review article by Jerri [11]) which is stated below as Theorem 1 .

\section{Theorem 1: The Uniform 1-D Sampling Theorem (WKS)}

If $f(t)$ is a function of one variable having a Fourier transform $F(\omega)$ such that $F(\omega)=0$ for $\omega \geq \omega_{0}=\pi / T$ and is sampled at the points $t_{n}=n T$, then $f(t)$ can be reconstructed exactly from its samples $f(n T)$ as follows:

$$
f(t)=\sum_{n=-\infty}^{\infty} f(n T) \sin \left[\omega_{0}(t-n T)\right] /\left[\omega_{0}(t-n T)\right]
$$

Let us now consider a nonuniform sample sequence $\left\{t_{n}\right\}$ where $t_{n}$, the position of the $n$th sample, is not necessarily equal to $n T$. For example, refer to the function shown in Fig. 1(a). This function is sampled nonuniformly as shown at the locations $t_{n}$. Now, suppose we apply a stretching/compressing transformation to $f(t)$, described by $\tau=\gamma(t)$, such that we end up with another function $h(\tau)$ as shown in Fig. 1(b), with a sampling period of $T$ units. If the transformation, $\gamma$, between $t$ and $\tau$ is such that $t_{0}+n T=\gamma\left(t_{n}\right)$, for some arbitrary $t_{0}$, then the samples of the function $h(\tau)$ will be uniformly spaced [Fig. 1 (b)] and we can use Theorem 1 . For the reconstruction of $h(\tau)$ to be exact we must have that $h(\tau)$ be band limited to $\omega_{0}$ 


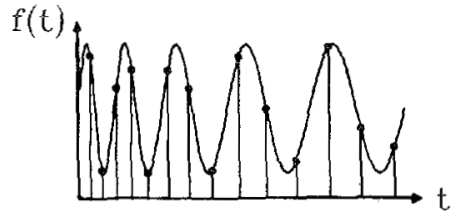

(a)

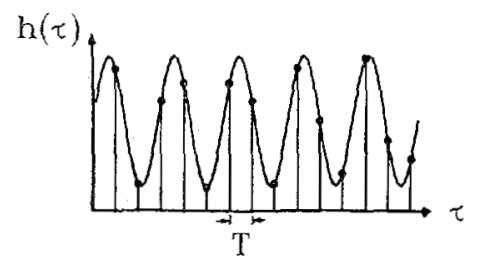

(b)

Fig. 1. (a) A function $f(t)$ sampled at nonuniformly distributed positions. (b) The transformed function $g(\tau)$, sampled at uniformly distributed positions.

$=\pi / T$. If this is so, we can then reverse the stretching/ compression operation, and retrieve the reconstructed version of the function $f(t)$ by using the relationship

$$
f(t)=h(\gamma(t)) \text {. }
$$

Substituting this relationship into (1) of Theorem 1, and using $\tau=\gamma(t)$ yields

$$
f(t)=\sum_{n=-\infty}^{\infty} f\left(t_{n}\right) \sin \left[\omega_{0}(\gamma(t)-n T)\right] /\left[\omega_{0}(\gamma(t)-n T)\right]
$$

Hence, in order to reconstruct $f(t)$ from its nonuniformly spaced samples $f\left(t_{n}\right)$, it suffices to find the invertible and oneto-one function $\gamma(t)$ such that $\gamma\left(t_{n}\right)=n T$ and then to use (3).

The reconstruction formula (3) is equivalent to the one derived by Papoulis [15], who treated the case of sample positions that were deviated slightly from a uniform sample distribution. However, his analysis indicated that this reconstruction would never be exact, but would always be subject to an aliasing error which became smaller as the sample deviation became smaller. This conclusion is too pessimistic, however, and it can be shown that there are cases for which the samples are not uniformly distributed and yet the reconstruction can be exact. The conditions under which an exact reconstruction can be obtained are discussed below.

In order for (3) to hold, the function $h(\tau)$ must be band limited to $\omega_{0}$. Thus $h(\tau)$ is a member of the set $B_{\omega_{0}}$, which is defined as the set of all functions whose Fourier transforms vanish for $|\omega|>\omega_{0}$. Let us define the set $C_{\gamma}$ to be the set of all functions which are the image of a function in $B_{\omega_{0}}$ under the transformation $\gamma^{-1}$. It can be seen that $C_{\gamma}$ is the set of all functions that can be reconstructed exactly with (3), for a given $\gamma$.

The set $C_{\gamma}$ is clearly nonempty, and thus Papoulis' assertion that (3) is only approximately true for all functions is incorrect. Equation (3) is approximate only for those functions that are not members of $C_{\gamma}$.

An interesting point to be noted is that the functions in $C_{\gamma}$ are generally not band limited. That this is so can be seen by examination of the relationship between the spectra of $h(\tau)$ and $f(t)$. It is possible to show that

$$
F(\lambda)=\int_{-\omega_{0}}^{\omega_{0}} P(\lambda, \omega) H(\omega) d \omega
$$

where the function $P$, which can be thought of as a frequencyvariant blurring function, is defined by

$$
P(\lambda, \omega)=\int_{-\infty}^{\infty} e^{j 2 \pi\left(\omega \gamma^{-1}(\tau)\right)} e^{-j 2 \lambda \tau} d \tau .
$$

That is $P(\lambda, \omega)$ is the Fourier transform of the angle modulated signal

$$
p_{\omega}(\tau)=e^{j 2 \pi \omega \gamma-1}{ }^{-1}(t) .
$$

Thus, if $p_{\omega}(t)$ is not band limited, as is usually the case for angle modulated signals, then generally $f(t)$ will not be either.

One can show that there exists a transformation $\gamma$ such that the FM signal defined by

$$
f(t)=e^{-j !_{0}^{t} \Omega(s) d s}
$$

is a member of $C_{\gamma}$ when $\Omega(s)$ is a positive, continuous function. Hence, such a function can always be reconstructed exactly, when sampled at the times $t_{n}=\gamma^{-1}(n T)$, even when it is not strictly band limited.

Let us summarize the details of the above analysis in the form of a theorem.

\section{Theorem 2: The Nonuniform 1-D Sampling Theorem}

Let a function $f(t)$ of one variable be sampled at the points $t$ $=t_{n}$, where $t_{n}$ is not necessarily a sequence of uniformly spaced numbers. If a one-to-one continuous mapping $\gamma(t)$ exists such that $n T=\gamma\left(t_{n}\right)$, and if $h(\tau)=f\left(\gamma^{-1}(\tau)\right)$ is band limited to $\omega_{0}=\pi / T$, then the following equation holds

$$
f(t)=\sum_{n=-\infty}^{\infty} f\left(t_{n}\right) \sin \left[\omega_{0}(\gamma(t)-n T)\right] /\left[\omega_{0}(\gamma(t)-n T)\right] .
$$

The reconstruction method described here can be thought of in a different manner. Consider a "burst" type signal such as that shown in Fig. 2. Intuitively, we would expect that a uniform sampling of $f(t)$ that allows an exact reconstruction would not be the most efficient sampling scheme. It seems reasonable to require a higher sample density in the center region where there are high frequency components than in the outer regions where there are lower frequency components. Suppose that, at every point of the function $f(t)$, we make a local estimate of its bandwidth-B(t). Then, it would follow that we would have a sample at a rate of $2 B(t)$ samples/unit time in order to allow an exact reconstruction of the signal. This conclusion has been reached (albeit from a different direction) by Horiuchi [10] who derived the reconstruction formula (10) for a signal with a time varying bandwidth $B(t)$ that is sampled at the points $t_{n}$ given implicitly by

$$
t_{n}=n /\left(2 B\left(t_{n}\right)\right)
$$

$$
f(t)=\sum_{n=-\infty}^{\infty} f\left(t_{n}\right) \sin [\pi(2 B(t) t-n)] /[\pi(2 B(t) t-n)] .
$$




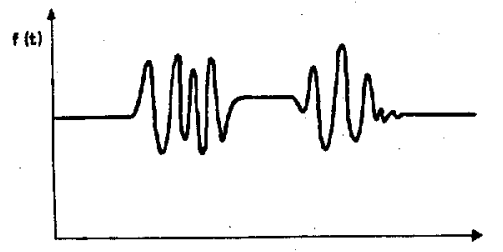

Fig. 2. A "burst" type of signal, with time-varying bandwidth.

The derivative of the mapping function $\partial \gamma(t) / \partial t$ can be thought of as the instantaneous sampling rate and hence we have that

$$
\partial \gamma(t) / \partial t=\left(2 \pi / \omega_{0}\right) B(t) \text { or } \gamma(t)=k+\int_{0}^{t}\left(2 \pi / \omega_{0}\right) B(r) d r
$$

If the bandwidth $B(t)$ is a constant (or approximately so over a given interval) then we can say

$$
\gamma(t)=\left(2 \pi / \omega_{0}\right) B(t) t .
$$

With this equation for $\gamma(t)$ we can see that (3) and (10) are equivalent. If the bandwidth is not approximately constant then our equation (3) and Horiuchi's equation (10) are not equivalent.

Equations (9) and (11) tells us (implicitly) how to optimally sample a signal, that is, how to sample a signal with the smallest number of sample points while still allowing an exact reconstruction of the signal. Equation (10) suggests that we could interpret the reconstructed $f(t)$ as the response of a time varying (or adaptive) low-pass filter, with bandwidth $B(t)$, to the signal $\sum_{n=-\infty}^{\infty} f(t) \delta\left(t-t_{n}\right)$. Thus one can envisage the following process. Given an arbitrary function $f(t)$ we estimate its bandwidth as a function of time. We then integrate this bandwidth function, as in (11), to yield the warping function $\gamma(t)$. When this warping function crosses an integer value- $n$, we sample $f(t)$. We then store or transmit the sample $f\left(t_{n}\right)$ along with the time at which the sample was taken, $t_{n}$. We can then, knowing all of the $f\left(t_{n}\right)$ and $t_{n}$ values, reconstruct $f(t)$ using (8).

The preceding analysis is complicated by the fact that an exact "local" bandwidth measure does not exist as bandwidth is defined globally, being a frequency domain measure. Thus, we can only obtain local bandwidth "estimates," which may cause concern as to the validity of (9)-(11). In practice, however, the reconstruction formulas that are used are defined only over a finite area (truncation of the reconstruction series), and so one loses nothing by assuming the local bandwidth over this finite area to be the actual bandwidth.

In practice, the use of the above reconstruction theorem requires the knowledge of the function $\gamma(t)$ at all points $t$ for which we desire a reconstruction. If an analytical expression for the sampling sequence $t_{n}$ is known [e.g., $t_{n}=s(n)$ ] then we can simply extend this analytical expression to include noninteger values [e.g., $\gamma(t)=s(t)$ ]. If no such analytical expression is available (as is usually the case) or if the analytical expression cannot be extended to noninteger values, then $\gamma(t)$ must be found by interpolation between the known $\gamma(n)$ points. The only constraint on this interpolation is that it must yield a $\gamma$ that is one-to-one and invertible (monotonic).

Let us now examine the extension of the above onedimensional sampling theory to the case of two dimensions.

\section{Two-Dimensional Sampling Theory}

As in the one-dimensional case, the development of a twodimensional nonuniform sampling theory begins with the consideration of the uniform sampling theory. The theory behind the reconstruction of functions of two variables from uniformly distributed samples of these functions was developed by Petersen and Middleton [16]. Mersereau [12] and Mersereau and Speake [13] have studied the more general problem of processing multidimensional signals that have been sampled on uniform lattices, especially hexagonal lattices which have added importance in this paper. We are concerned here only with signal reconstruction but it is evident that the type of signal processing techniques described by Mersereau and Speake can be extended, using the results of this paper, to the case of nonuniform sampling.

The essentials of the work of Petersen and Middleton [16] is summarized in Theorem 3. This theorem describes the conditions under which a function of two variables, $f(\bar{x})$, can be reconstructed exactly from its samples taken at points on a uniform lattice. This theorem basically extends the onedimensional uniform sampling theorem (Theorem 1) to two dimensions.

\section{Theorem 3: The Uniform Two-Dimensional Sampling Theorem}

Suppose that a function of two variables $f(\bar{x})$ is sampled at points in the infinite sampling set $\left\{\bar{x}_{s}\right\}$ defined by

$$
\begin{aligned}
\bar{x}_{s}=\left\{\bar{x}: \bar{x}=1_{1} \bar{v}_{1}+1_{2} \bar{v}_{2}, 1_{1}, 1_{2}=0, \pm 1,\right. & \pm 2, \pm 3 \cdots \\
\cdot & \left.\bar{v}_{1} \neq k \bar{v}_{2}\right\}
\end{aligned}
$$

The vectors $\bar{v}_{1}$ and $\bar{v}_{2}$ form the basis for the sampling lattice defined by the points in $\left\{\bar{x}_{s}\right\}$. Such a sampling lattice is shown in Fig. 3 for $\bar{v}_{1}=\left(2 / \sqrt{3,1}\right.$ and $\bar{v}_{2}=(1 / \sqrt{3,1})$. Furthermore, let the support of the Fourier transform $F(\bar{\omega})$ of $f(\bar{x})$ be bounded by the region $R$ in $\bar{\omega}$ space. The spectrum of the sampled function, $f_{s}(\bar{x})=\Sigma_{\left\{\bar{x}_{s}\right\}} \delta\left(\bar{x}-\bar{x}_{s}\right) f(\bar{x})$, is made up of an infinite number of repetitions of the spectrum $F(\bar{\omega})$ and is given by $F_{s}(\bar{\omega})=\Sigma \Sigma_{\left\{\bar{\omega}_{s}\right\}} F\left(\bar{\omega}+\bar{\omega}_{s}\right)$, where the set $\left\{\bar{\omega}_{s}\right\}$ is defined by

$$
\begin{array}{r}
\bar{\omega}_{s}=\left\{\bar{\omega}: \bar{\omega}=1_{1} \bar{u}_{1}+1_{2} \bar{u}_{2}, 1_{1}, 1_{2}=0, \pm 1, \pm 2, \pm 3 \cdots,\right. \\
\left.\cdot \bar{u}_{1} \neq k \bar{u}_{2}\right\}
\end{array}
$$

and where the frequency domain basis vectors $\bar{u}_{1}$ and $\bar{u}_{2}$ are related to the spatial domain basis vectors $\bar{v}_{1}$ and $\bar{v}_{2}$ by

$$
\bar{u}_{1}^{T} \bar{v}_{1}=\bar{u}_{2}^{T} \tilde{v}_{2}=2 \pi, \text { and } \tilde{u}_{1}^{T} \bar{v}_{2}=\ddot{u}_{2}^{T} \bar{v}_{1}=0 .
$$

If $F(\bar{\omega})=0$ when $F\left(\bar{\omega}+\bar{\omega}_{s}\right) \neq 0$ (for every $\bar{\omega}_{s} \neq 0$ ), then the spectral repetitions do not overlap, and the following equation holds

$$
f(\bar{x})=\sum_{\left\{\bar{s}_{s}\right\}} f\left(\bar{x}_{s}\right) g\left(\bar{x}-\bar{x}_{s}\right)
$$




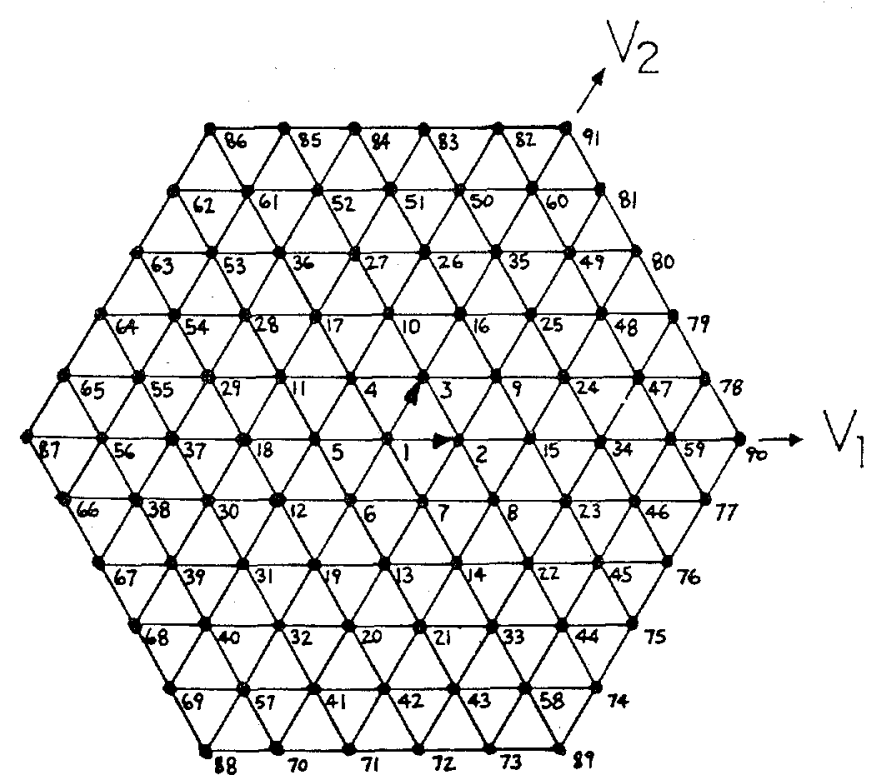

Fig. 3. The hexagonal sampling lattice for functions with isotropic spectra.

where $g(\bar{x})$ is the inverse Fourier transform of the low-pass filter function $G(\bar{\omega})$ defined by

$$
\begin{array}{ccc}
=Q & \bar{\omega} \in R \\
G(\bar{\omega})=\text { arbitrary } & \bar{\omega} \notin R, \bar{\omega}-\bar{\omega}_{s} \notin R \\
=0 & \bar{\omega}-\bar{\omega}_{s} \in R .
\end{array}
$$

$Q$ is a constant that is equal to the area of each of the sampling lattice cells and is the inverse of the sample density. In terms of the sampling lattice basis vectors, $\bar{v}_{1}$ and $\bar{v}_{2}, Q$ is given by

$$
Q=\sqrt{ }\left|\bar{v}_{1}\right|^{2}\left|\bar{v}_{2}\right|^{2}-\left(\bar{v}_{1}^{T} \bar{v}_{2}\right)^{2} \text {. }
$$

Now, following the lead of the analysis performed in Section II for the one-dimensional case, let us introduce a second function of two variables, $h(\bar{x})$, that is the image of $f(\bar{x})$ under the coordinate transformation

$$
\bar{\xi}=\bar{\gamma}(\bar{x})
$$

i.e.,

$$
f(\bar{x})=h(\bar{\xi})=h(\bar{\gamma}(\bar{x})) .
$$

Let the coordinate transformation, $\bar{\gamma}(\bar{x})$, be such that the set of nonuniformly spaced samples $\left\{\bar{x}_{s}\right\}$, is transformed into a uniformly spaced set of samples $\left\{\vec{\xi}_{s}\right\}$ [such as the set defined by (13)]. Since $\left\{\bar{\xi}_{s}\right\}$ contains points that lie on a regular lattice, the function $h(\bar{\xi})$ can be reconstructed under the conditions of Theorem 3 . If $h(\bar{\xi})$ is suitably band limited then this reconstruction will be exact. Once $h(\bar{\xi})$ has been reconstructed, $f(\bar{x})$ can be obtained by reversing the coordinate transformation (19). This is the basis of our nonuniform two-dimensional sampling theorem which is stated below as Theorem 4.

\section{Theorem 4: The Nonuniform Two-Dimensional Sampling Theorem}

Suppose that a function of two variables $f(\bar{x})$ is sampled at points in the infinite set $\left\{\bar{x}_{s}\right\}$. Now, if there exists a one-to-one continuous mapping $\bar{\gamma}$ such that

$$
\bar{\xi}=\bar{\gamma}(\bar{x}) \text { and } \bar{\xi}_{s}=\bar{\gamma}\left(\bar{x}_{s}\right)
$$

and if the function defined by

$$
h(\bar{\xi})=f\left(\bar{\gamma}^{-1}(\bar{\xi})\right)
$$

satisfies the conditions of Theorem 3 then the following is true:

$$
h(\bar{\xi})=\sum_{\left\{\bar{\xi}_{s}\right\}} h\left(\bar{\xi}_{s}\right) g\left(\bar{\xi}-\bar{\xi}_{s}\right)
$$

where $g(\xi)$ is as defined by (17). Hence

$$
f(\bar{x})=\sum_{\left\{\bar{x}_{s}\right\}} f\left(\bar{x}_{s}\right) g\left(\bar{\gamma}(\bar{x})-\bar{\gamma}\left(\bar{x}_{s}\right)\right) .
$$

Let us assume that $h(\bar{\xi})$ is an isotropic function, where we have here taken the term isotropic, as in [16], to mean that the support of the Fourier transform of the function is a disk shaped region in the frequency plane, centred about the origin. If this is the case, we can define the region $R$, mentioned in Theorem 3, as $R=\{\bar{\lambda}:|\bar{\lambda}| \leq \pi\}$. It can then be shown that ([16], (74), with $B=1 / 2$ ):

$$
g(\bar{\xi})=(\pi / \sqrt{ } 3) J_{1}(\pi|\bar{\xi}|) /(\pi|\bar{\xi}|)
$$

where $J_{1}$ is the first-order Bessel function of the first kind. Combining (24) and (25) yields the following result:

$$
\begin{aligned}
f(\bar{x})=\sum_{\left\{x_{s}\right\}} f\left(\bar{x}_{s}\right)(\pi / \sqrt{ } 3) J_{1}(\pi \mid \bar{\gamma}(\bar{x})- & \left.\bar{\gamma}\left(\bar{x}_{s}\right) \mid\right) \\
\cdot & \cdot /\left(\pi\left|\bar{\gamma}(\bar{x})-\bar{\gamma}\left(\bar{x}_{s}\right)\right|\right) .
\end{aligned}
$$

It can be shown [16] that the most efficient sampling lattice (in $\bar{\xi}$ space) for such an isotropic process is a hexagonal lattice with a characteristic spacing of $2 / \sqrt{3}$. This sampling lattice is the one shown in Fig. 3. The values of $\bar{\xi}_{s}$ are fixed by this lattice and, by (25), the values of $\bar{\gamma}\left(\bar{x}_{s}\right)$ are also fixed.

\section{Determination of $\bar{\gamma}(\bar{x})$}

If we are to use (24) as our reconstruction formula, we must, as in the one-dimensional case, know the mapping function $\bar{\gamma}(\bar{x})$ at all sample points and at all other points at which to obtain a reconstruction. As in the one-dimensional case we can, once we know the values of $\bar{\gamma}(\bar{x})$ at the sample points $\left\{\bar{x}_{s}\right\}$, interpolate to find $\bar{\gamma}(\bar{x})$ for any $\bar{x}$. Unlike the onedimensional case, however, it is not a trivial matter to obtain a mapping, $\Gamma:\left\{\bar{x}_{s}\right\}->\left\{\bar{\xi}_{s}\right\}$, between the sample sets in $\bar{x}$ and $\bar{\xi}$ space, that yields a one-to-one and continuous mapping function $\gamma$. In the one-dimensional case one and only one such mapping exists (restricting the sign of the derivative of $\gamma$ to be positive), given by interpolation of $\gamma\left(t_{n}\right)=n T$, but in two dimensions there may be, in general, any number of such mappings. The difficulty lies in the fact that there is no general scheme for ordering arbitrarily distributed points in two dimensions analogous to the sequential ordering available in one dimension, such that adjacency properties are preserved.

For the purpose of the following discussion let us make the following definitions. 
Definition: Partition-A partition of a planar region $R$ is a set of line segments, called links, that divide $R$ into a number of distinct, possibly overlapping subregions. The endpoints of these links are called the vertices of the partition. There can be no free vertices (i.e., those vertices belonging to only one link) in a partition except at the boundary of $R$.

Definition: Tessellation-A tessellation is a partition whose regions do not overlap.

Definition: Voronoi Tessellation-The Voronoi tessellation of the $\bar{\xi}$ plane with respect to the point set $\left\{\xi_{s}\right\}$ is the tessellation whose links consist of points equidistant from any two points $\bar{\xi}_{i}, \bar{\xi}_{j} \in\left\{\bar{\xi}_{s}\right\}$ and no closer to any other points $\bar{\xi}_{k}$ $\in\left\{\bar{\xi}_{s}\right\}$. The vertices of the Voronoi tessellation are those points equidistant from three or more points in $\left\{\xi_{s}\right\}$ and no closer to any other point in $\left\{\bar{\xi}_{s}\right\}$. The subregions created by the Voronoi tessellation contain all points $\bar{\xi}$ closer to a given point in $\left\{\bar{\xi}_{s}\right\}$ than any other point in $\left\{\bar{\xi}_{s}\right\}$.

Definition: Dirichlet Tessellation-The Dirichlet tessellation (sometimes referred to as the Delaunay triangulation) is the dual of the Voronoi tesselation. The Dirichlet tessellation can be thought of as the set of line segments connecting the points in $\left\{\bar{\xi}_{s}\right\}$ whose subregions in the Voronoi tessellation of $\bar{\xi}$ with respect to $\left\{\bar{\xi}_{s}\right\}$ share a common link.

An example of Voronoi and Dirichlet tessellations are shown in Fig. 4. Further discussion of Dirichlet and Voronoi tessellations can be found in Ahuja and Schacter [2].

It can be seen that the partition created by connecting the points in the hexagonal sampling lattice to their nearest neighbours is a Dirichlet tessellation and as such, the regions created by the partition are nonoverlapping triangles. We will denote this particular tessellation by $D_{h}$.

Definition: $P$-Adjacency - Two points are defined to be $P$ adjacent if they are vertices of a partition $P$ and share a common link.

Definition: Adjacency Conserving Partition Mapping (ACPM)-A mapping, $\Gamma:\left\{\bar{x}_{s}\right\}->\left\{\bar{\xi}_{s}\right\}$, is termed an ACPM if it takes a partition having vertex set $\left\{\bar{x}_{s}\right\}$ into a partition having vertex set $\left\{\bar{\xi}_{s}\right\}$ such that the points in $\left\{\bar{x}_{s}\right\}$ have the same $P$-adjacency as their images in $\left\{\xi_{s}\right\}$.

As a result of the preservation of adjacency properties, a region in a partition has the same number of links as the corresponding region in its image under an ACPM. Also, each vertex has the same number of links as its image under an ACPM. Hence, since $D_{h}$ has triangular regions, the regions of any ACPM, $P_{x}$, of $D_{h}$ are also triangular (although possibly overlapping). Note also that the inverse of an ACPM is itself an ACPM.

Definition: Generalized Hexagonal Tessellation $(G H T)$ - A tessellation created by applying an ACPM to the tessellation $D_{h}$ is called a generalized hexagonal tessellation, or GHT. All vertices of a GHT are the junction of six links.

As we said at the beginning of this section, once we have a mapping, $\Gamma$, between the points in $\left\{\bar{x}_{s}\right\}$ and the points in $\left\{\bar{\xi}_{s}\right\}$, we can determine the mapping function, $\bar{\gamma}(\bar{x})$, for $\bar{x}$ not necessarily a member of $\left\{\bar{x}_{s}\right\}$, by interpolation of the $\Gamma$ values.

The fact that the regions of $P_{x}$ are triangular suggests that, given a point $\bar{x}$ in the interior of one of these regions, we

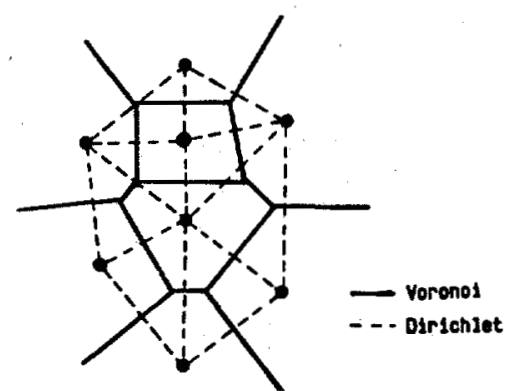

Fig. 4. The Voronoi and Dirichlet tessellations for a set of points.

should let $\bar{\xi}=\bar{\gamma}(\bar{x})$ be some linear combination of the (known) values of $\bar{\gamma}(\bar{x})$ at the vertices of this region. Such a linear combination can be written as

$$
\bar{\gamma}(\bar{x})=\sum_{x_{i} \in V(x)} \Gamma\left(\bar{x}_{i}\right) I\left(\bar{x}, \bar{x}_{\left(i_{3}\right.}, \bar{x}_{(i+1)_{3}}, \bar{x}_{(i+2)_{3}}\right)
$$

where $V(\bar{x})=\left(\bar{x}_{1}, \bar{x}_{2}, \bar{x}_{3}\right)$ is the vertex set of the region of $P_{x}$ containing $\bar{x}$ and where $(i)_{3}$ denotes $(i)$ modulo $(3)+1$. The function $I\left(\bar{x}, \bar{x}_{(i)_{3}}, \bar{x}_{(i+1)_{3}}, \bar{x}_{(i+2)_{3}}\right)$ is some interpolation function which results in an invertible $\bar{\gamma}$.

The simplest such interpolation that we can do between three noncollinear points is a trilinear interpolation which fits a planar (vector valued) surface to these three points. The interpolation function for this method is given in (28). This equation describes a plane passing through the points $\left(x_{1}^{1}, x_{1}^{2}\right.$, $1),\left(x_{2}^{1}, x_{2}^{2}, 0\right)$, and $\left(x_{3}^{1}, x_{3}^{2}, 0\right)$.

$$
\begin{aligned}
I\left(\bar{x}, \bar{x}_{1}, \bar{x}_{2}, \bar{x}_{3}\right)=[ & x^{1}\left(x_{2}^{2}-x_{3}^{2}\right)+x^{2}\left(x_{3}^{1}-x_{2}^{1}\right) \\
& \left.+\left(x_{3}^{2} x_{2}^{1}-x_{2}^{2} x_{3}^{1}\right)\right] / \Delta
\end{aligned}
$$

where

$$
\Delta=x_{1}^{1}\left(x_{2}^{2}-x_{3}^{2}\right)-x_{2}^{1}\left(x_{2}^{1}-x_{3}^{1}\right)+\left(x_{2}^{1} x_{3}^{2}-x_{3}^{1} x_{2}^{2}\right)
$$

and $\bar{x}=\left(x^{1}, x^{2}\right)$.

We can now state the following theorem which supplies the conditions under which a given one-to-one point set mapping will yield the one-to-one and continuous mapping (transformation) function that is required for (24) to be valid.

\section{Theorem 5: Invertibility of a Mapping}

Given the Dirichlet tessellation $D_{h}$ on $\bar{\xi}$ as defined above, and the trilinear interpolation defined by (28), then, if there is an ACPM, $\Gamma^{-1}$, such that the image of $D_{h}-P_{x}$ is a GHT and the points in the set $V(\bar{x})$ are not collinear, the mapping $\bar{\gamma}(\bar{x})$ defined by (27) is one-to-one and continuous.

The key condition in Theorem 5 is that $\Gamma^{-1}$ be an ACPM, or conversely, that $P_{x}$, the image of $D_{h}$, is a GHT. Thus, in order to perform the reconstruction of a function for a given sample set $\left\{\bar{x}_{s}\right\}$, we need to first find the tessellation $P_{x}$, whose image under the mapping $\Gamma$ is the hexagonal lattice tessellation $D_{h}$. The regions of $P_{x}$ all must have three sides and the vertices of $P_{x}$ must be the junction of six links.

It is suspected that it is not possible to create a GHT from all sets of points $\left\{\bar{x}_{s}\right\}$. The authors have no proof of this conjecture, but it can be seen that algorithms for creating GHT's run into trouble trying to order large numbers of points in some cases. For example, consider the set of points shown 


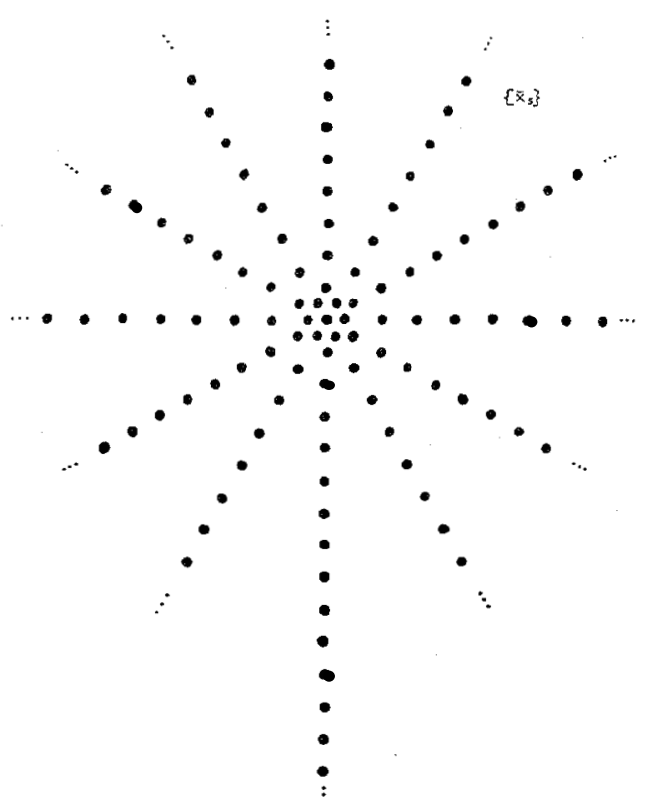

(a)

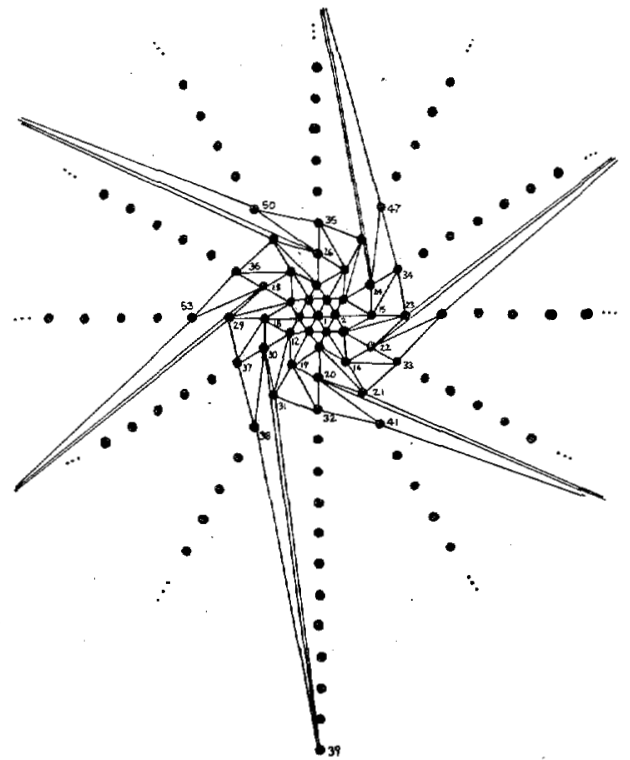

(b) :

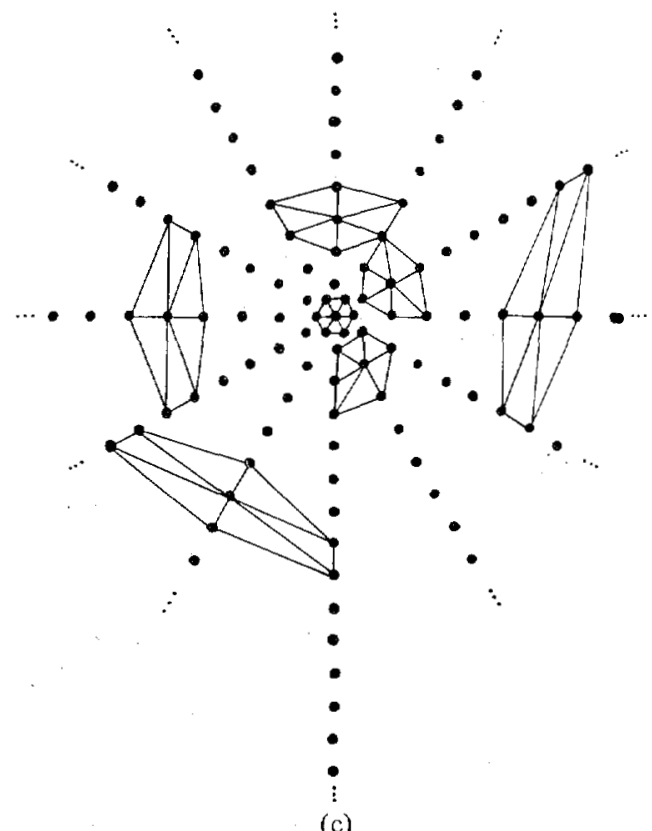

Fig. 5. (a) A set $\left\{\bar{x}_{s}\right\}$ made up of points along twelve radial lines. (b) An attempt to create a GHT made up of the points in $\left\{\bar{x}_{s}\right\}$. The attempt fails after mapping a certain number of points. (c) Some local GHT's of the point set of $5(a)$.

in Fig. 5(a). This set made up of points that lie along twelve radial lines. This type of sample set is found in $X$-ray tomography (e.g., see Fig. 3 of [14]). We try to create a GHT from this set by trying to map points in it to $D_{h}$ by following the ordering of points in $\left\{\bar{\xi}_{s}\right\}$ given in Fig. 3. The result of this process is shown in Fig. 5(b). As we proceed the regions of the tessellation become thinner and less regular. It turns out that, using our construction, beyond a certain point no points can be mapped without creating an overlapping region or a region with collinear vertices. This, of course, does not constitute a valid proof of our conjecture as there are a number of other ways of trying to construct a GHT from this set of points, one of which may work. However, this example does point out the problems involved in performing the mapping operation. Even if a GHT could be found the thinness of the regions of the GHT so found would cause problems with the interpolation process.

However, if we truncate the reconstruction formula (24) to only take into account those samples in a finite region about $\bar{x}$, then it would not be necessary for $\bar{\gamma}$ to be one-to-one and continuous everywhere. The mapping function need be one-toone and continuous only over this restricted region. This would mean that we would need only to find a mapping function that created a partition that was only locally a GHT 
In this way it is expected that a mapping could be found for any set $\left\{\bar{x}_{s}\right\}$. For example, Fig. 5(c) shows a few of these local GHT's defined on the sample set of Fig. 5(a). The price we pay for this weakening of Theorem 4 is that the reconstruction is no longer exact, even if $h(\bar{\xi})$ is suitably band limited. In practice such a truncation of the reconstruction equation is unavoidable as one can only process a finite number of samples in a finite time.

Let the finite set of sample points used to reconstruct $f(\bar{x})$ be $\left\{\bar{x}_{s}\right\}_{0} \in\left\{\bar{x}_{s}\right\}$. Note that for different reconstruction points $\bar{x}$ we may have different sets $\left\{\bar{x}_{s}\right\}_{0}$. When only a finite number of terms are used in (24) the resulting value of $f\left(\bar{x}_{0}\right)$ will not be exact but will be subject to a "truncation" error term. This truncation error is defined in (30) and can be bounded as shown in (31).

$$
\begin{aligned}
e_{t}^{2}(\bar{x}) & =\left|f(\bar{x})-f_{R}(\bar{x})\right|^{2} \\
& =\left|\sum_{\left\{\bar{s}_{s}\right\}-\left\{\bar{x}_{s}\right\}_{0}} f\left(\bar{x}_{s}\right) g\left(\left|\bar{\gamma}(\bar{x})-\bar{\gamma}\left(\bar{x}_{s}\right)\right|\right) /\left(\pi\left|\bar{\gamma}(\bar{x})-\bar{\gamma}\left(\bar{x}_{s}\right)\right|\right)\right|^{2} \\
& \leq f_{\max }^{2} \sum_{\left\{\bar{s}_{s}\right\}-\left\{\xi_{s}\right\}_{0}} 1 /\left(2 \pi\left|\bar{\xi}-\bar{\xi}_{s}\right|^{2}\right) .
\end{aligned}
$$

where $g(x)$ is as in (25) and in finding this bound we have used the Triangle Inequality and the fact that $\left|J_{1}(x)\right| \leq 1 / \sqrt{ } 2[1]$.

The above bound suggests that we make the distances between $\bar{\xi}$ and all points not in $\left\{\bar{\xi}_{s}\right\}_{0}$ as large as possible for $\bar{\xi}$ $\in\left\{\bar{\xi}_{s}\right\}_{0}$. In other words, $\left\{\bar{\xi}_{s}\right\}_{0}$ should consist of the $N_{s}$ points closest to $\bar{\xi}$.

How are we to determine which points $\left\{\bar{x}_{s}\right\}_{0}$ map into $\left\{\bar{\xi}_{s}\right\}_{0}$ ? Theorem 5 provides one constraint on $\left\{\bar{x}_{s}\right\}_{0}$ by requiring that the partition $P_{x 0}$ be a tessellation and map continuously into the Dirichlet tessellation $D_{h 0}$, where $P_{x 0}$ and $D_{h 0}$ are the partitions formed from the points in $\left\{\bar{x}_{s}\right\}_{0}$ and $\left\{\bar{\xi}_{s}\right\}_{0}$ respectively.

In order for the interpolation of $\bar{\gamma}$ [equation (27)] to be valid we must stipulate that $\bar{x}$ be contained in one of the regions of $P_{x 0}$. This also means that $\bar{\xi}$ lies in one of the regions of $\left\{\xi_{s}\right\}_{0}$.

Theorem 4 requires that $H(\bar{\lambda})=0$ when $H\left(\bar{\lambda}+\bar{\lambda}_{s}\right) \neq 0$ for every $\bar{\lambda}_{s} \neq 0$. If this condition is not satisfied the value of $f(\bar{x})$ obtained with (24) will be in error. This error is referred to as aliasing error. It can be shown that if the aliasing errors due to two different sample distributions are compared, the distribution with the higher density will have the lower aliasing error. Now, it can shown that, for a given $f(\bar{x})$, the localized aliasing error of the reconstruction (24) decreases as the density of $\left\{\bar{x}_{s}\right\}_{0}$ increases. This suggests that we should select $\left\{\bar{x}_{s}\right\}_{0}$ that has, in addition to the above mentioned conditions, a maximum possible density. This will ensure that, for a given $f(\bar{x})$, we will obtain a minimum possible aliasing error, or, alternatively, will give us the maximum allowable band limit that a function can possess while still yielding an exact reconstruction.

We can summarize the conditions on $\left\{\bar{x}_{s}\right\}_{0}$ as follows:

1) The set $\left\{\bar{x}_{s}\right\}_{0}$ must be such that there exists a tessellation $P_{x 0}$ that can be continously mapped into the Dirichlet tessellation $D_{h 0}$.

2) The point $\bar{x}$ at which the function is to be reconstructed must lie within one of the regions of $P_{x 0}$.
3) The density of the set $\left\{\bar{x}_{s}\right\}_{0}$ must be the maximum possible subject to the above two constraints.

In general, finding the optimal mapping that jointly minimizes the aliasing and truncation errors is very difficult. In the next section we will present a heuristic algorithm which is near optimal for homogenous sample distributions. This algorithm guarantees finding a mapping which locally satisfies the conditions of Theorem 5 .

\section{A Reconstruction Algorithm}

Based on the discussions in Sections III and IV we propose the following reconstruction algorithm. This algorithm finds, for a given point $\bar{x}$ and sample set $\left\{\bar{x}_{s}\right\}$, a subset $\left\{\bar{x}_{s}\right\}_{0}$ of $\left\{\bar{x}_{s}\right\}$ that locally satisfies the conditions of Theorem 5 . It will be seen that this algorithm is generally suboptimal, in that the truncation and aliasing errors may not be the minimum possible value. For homogenous sample distributions, however, this algorithm will be optimal.

The motivation behind the algorithm is as follows. In the application that initiated this study, the sample distributions were nonuniform but homogenous. This is the case in many applications. Thus the sample points $\left\{\bar{x}_{s}\right\}$ could be thought of arising from the perturbation of a regular sample set, such as the hexagonal lattice. Our algorithm assumes that this perturbation is small enough so that a given point on the original hexagonal lattice will remain somewhere in a $60^{\circ}$ sector about its original position. See Fig. 6 for an example of such a perturbation.

The algorithm begins by trying to find the centre point of the "original" hexagonal lattice. Such a point is denoted by $\bar{\xi}_{0}$ in Fig. 7. We take as the perturbed value of this point, the point $\bar{x}_{0}$ in $\left\{\bar{x}_{s}\right\}$ closest to $\bar{x}$, the point at which we wish to obtain a reconstruction. This point will then be mapped to $\bar{\xi}_{0}$. In the particular algorithm described here we use $N_{s}$ equal to 7 . Algorithms for higher $N_{s}$ values can be devised but they become increasingly more difficult. Once we have the point $\bar{x}_{0}$ which maps into $\vec{\xi}_{0}$, we must find the other $N_{s}-1$ points of $\left\{\bar{x}_{s}\right\}$. Because we have assumed that the points in $\left\{\bar{x}_{s}\right\}$ result from the slight perturbations of the points in $\left\{\xi_{s}\right\}$ within $60^{\circ}$ sectors, and because we want $\left\{\bar{\xi}_{s}\right\}_{0}$ to consist of the $N_{s}$ points closest to $\bar{\xi}$, we can use the following heuristic procedure for determining $\left\{\bar{x}_{s}\right\}_{0}$. Divide the space in $\bar{x}$ about the point $\bar{x}_{0}$ into six regions, each consisting of a $60^{\circ}$ sector, as shown in Fig. 6. Find the point in each of these sectors closest to $\bar{x}_{0}$. These points will then be the 6 other points in $\left\{\bar{x}_{s}\right\}_{0}$. These points are mapped to $\left\{\bar{\xi}_{s}\right\}_{0}$ as shown in Fig. 7.

This algorithm is described procedurally in a pseudo high level language below:

procedure RECONSTRUCT $\left(\left\{\bar{x}_{s}\right\},\left\{f\left(\bar{x}_{s}\right)\right\}, \bar{x}, f(\bar{x})\right)$.

(* To reconstruct the value of a function $f(\bar{x})$ at a point $\bar{x}$ given an arbitrary set of function samples $\left\{\bar{x}_{s}\right\}$. It is assumed that $N_{s}=7$ and that $\left\{\bar{x}_{s}\right\}$ is homogenous. ${ }^{*}$ )

begin

IF $\bar{x}=\bar{x}_{i} \in\left\{\bar{x}_{s}\right\}$ THEN

ELSE

$$
f(\bar{x})=f\left(\bar{x}_{i}\right)
$$

StartSearch $=\bar{x}$ 


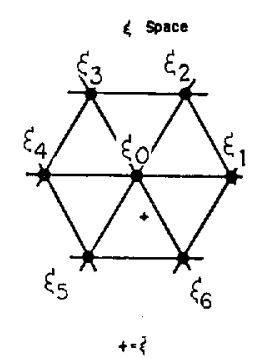

Fig. 6. The operation of the mapping heuristic for $N_{s}=7$.

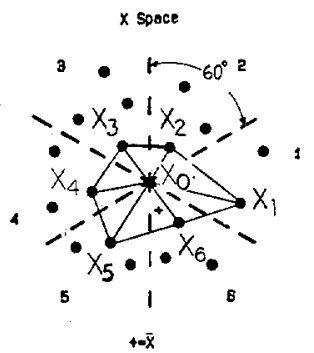

Fig. 7. The sample locations in $\bar{\xi}$ for $N_{s}=7$.

(* Start the spiral search at $x *$ )

FindNearestNeighbor $\left(\bar{x}_{0},\left\{\bar{x}_{s}\right\}, \bar{x}_{c}\right.$,

StartSearch)

$\left({ }^{*}\right.$ Look for the centre point of $\left.\left\{\bar{x}_{s}\right\}_{0}^{*}\right)$

FindMapping $\left(\bar{x}_{0},\left\{\bar{x}_{s}\right\},\left\{\bar{x}_{s}\right\}_{0},\left\{\bar{\xi}_{s}\right\}_{0}\right)$

(* Find the mapping between $\left\{\bar{x}_{s}\right\}_{0}$ and

$\left.\left\{\bar{\xi}_{s}\right\}_{0} *\right)$

InterpolateMapping $\left(\bar{x}_{0}, \bar{\xi}_{0},\left\{\bar{x}_{s}\right\}_{0},\left\{\bar{\xi}_{s}\right\}_{0}\right)$

(* Interpolate to find the mapping of $\bar{x}$ into $\bar{\xi}$

(e.g., using equations (27) and 28)) *)

$f(\bar{x})=0$

FOR $i=1$ TO $N_{s}$ DO

BEGIN

ENDFOR

( ${ }^{*}$ Compute the reconstruction sum $*$ )

$f\left(\bar{x}=f(\bar{x})+g\left(\bar{\xi}-\bar{\xi}_{i}\right) f\left(\bar{x}_{i}\right)\right.$

\section{ENDELSE}

endproc.

The nearest neighbor finding procedure "FindNearestNeighbor" can be done in a number of ways. For example, the efficient spiral search technique of [6], modified to search over monotonically increasing distances [4] was used in the examples described later in this paper.

The mapping procedures "FindMapping" determines the mapping between $\left\{\bar{x}_{s}\right\}_{0}$ and $\left\{\bar{\xi}_{s}\right\}_{0}$ given the sample set $\left\{\bar{x}_{s}\right\}$. This procedure is detailed in the following pseudo high level program.

$$
\begin{aligned}
& \text { procedure FindMapping }\left(\bar{x}_{0},\left\{\bar{x}_{s}\right\},\left\{\bar{x}_{s}\right\}_{0},\left\{\bar{\xi}_{s}\right\}_{0}\right) \\
& \bar{\xi}_{0}=(0,0) \\
& \text { StartSearch }=\bar{x}_{0} \\
& \left(* \text { Map } \bar{x}_{0} \text { to } \bar{\xi}_{0} \text {, the centroid of }\left\{\bar{\xi}_{s}\right\}_{0} *\right)
\end{aligned}
$$

while Found $1=$ false or Found $2=$ false or Found $3=$ false or Found $4=$ false or Found $5=$ false or Found $6=$ false do (* While the $N_{s}$ points in $\left\{\bar{\xi}_{s}\right\}_{0}$ have not all been assigned, do the following: *) begin

FindNearestNeighbor $\left(\bar{x}_{n},\left\{\bar{x}_{s}\right\}, \bar{x}_{0}\right.$, StartSearch)

(* Perform a spiral search, starting from StartSearch to find $\bar{x}_{n}$ closest to $\bar{x}_{0}$ but no nearer than StartSearch. *) If $\left(-30^{\circ} \leq \operatorname{Angle}\left(\bar{x}, \quad \bar{x}_{0}\right)<30^{\circ}\right)$ and $($ Found $1=$ false $)$ then

(* Determine whether or not $\bar{x}_{n}$ is in the $\bar{\xi}_{1}$ sector. If so assign $\bar{x}_{n}$ to $\bar{\xi}_{2} .{ }^{*}$ )

$\bar{\xi}_{1}=(2,2 / \sqrt{3)}$

$\bar{x}_{1}=\bar{x}_{n}$

Found $1=$ true

endif

If $\left(30^{\circ} \leq \operatorname{Angle}\left(\bar{x}, \bar{x}_{0}\right)\right.$ and $($ Found $2=$ false $)$

then

$\bar{\xi}_{2}=(1 / \sqrt{3,1})$

$\bar{x}_{2}=\bar{x}_{n}$

endif

Found $2=$ true

If $\left(90^{\circ} \leq \operatorname{Angle}\left(\bar{x}, \bar{x}_{0}\right)<150^{\circ}\right)$ and $($ Found $3=$ false $)$

then

$\xi_{3}=(-1 / \sqrt{3,1})$

Found $3=$ true

$\bar{x}_{3}=\bar{x}_{n}$

endif

If $\left(150^{\circ} \leq \operatorname{Angle}\left(\bar{x}, \bar{x}_{0}\right)<210^{\circ}\right)$ and $($ Found $4=$ false $)$

then

$$
\bar{\xi}_{4}=(-2 / \sqrt{3,0})
$$

Found $4=$ true

$\bar{x}_{4}=\bar{x}_{n}$

endif

If $\left(210^{\circ} \leq \operatorname{Angle}\left(\bar{x}, \bar{x}_{0}\right)<270^{\circ}\right)$ and $($ Found $5=$ false $)$

then

$$
\bar{\xi}_{s}=(-1 / \sqrt{3,-1})
$$

Found $5=$ true

$\bar{x}_{5}=\bar{x}_{n}$

endif

If $\left(270^{\circ} \leq \operatorname{Angle}\left(\bar{x}, \bar{x}_{0}\right)<330^{\circ}\right)$ and $($ Found $6=$ false $)$

then

$$
\bar{\xi}_{6}=(1 / \sqrt{ } 3,-1)
$$

Found $6=$ true

$$
\bar{x}, \bar{x}_{n}
$$

endif

StartSearch $=\bar{x}_{n}$

(*Start search for the next sample at $\bar{x}_{n}$ not $\bar{x}_{c} *$ )

\section{endwhile}

endproc.

The angle function used here computes the angle between the vector $\bar{x}-\bar{x}_{0}$ and some reference vector.

The above mapping heuristic works well when the samples are distributed more or less isotropically. For example, in Fig. $8(\mathrm{a})$, the set $\left\{\bar{x}_{s}\right\}_{0}$ of maximum density has been found. When the sample distribution is markedly nonisotropic it would be expected that another mapping procedure could do better, as can be seen in Fig. 8(b), where the optimum $\left\{\bar{x}_{s}\right\}_{0}$ has clearly not been found by the algorithm.

It is natural to ask how the reconstruction method described in this paper for one and two dimensions can be extended to 


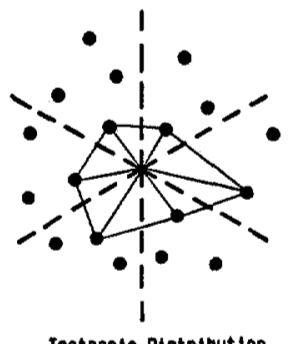

(a)

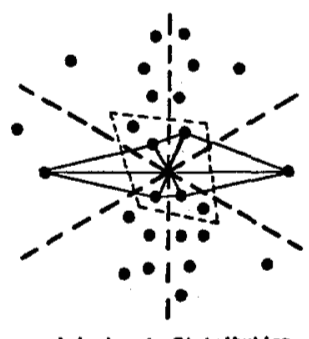

(b)

Fig. 8. The relation of the heuristic mapping efficiency in terms of sample density to the shape of the sample distribution.

the reconstruction of higher dimensional functions. It is evident that Theorem 4 , following the analysis of Petersen and Middleton [16] for the uniform case, can be directly extended to higher dimensionality, merely by increasing the dimensionality of the functions and variables involved. The search procedure, outlined above, can be similarly extended, to allow the determination of $\Gamma$ for any dimension. From this $\Gamma$, the function $\bar{\gamma}$ can be determined by fitting an $n$-dimensional hyperplane to $n+1$ points, where $n$ is the dimensionality of the sampled function. The efficiency of the mapping heuristic can be expected to fall as the dimension increases, however.

\section{EXAMPLeS}

In this section we provide examples of the application of the nonuniform sampling theory developed in this paper to the reconstruction of one- and two-dimensional functions.

\section{One Dimension}

In this example we examine the reconstruction of a sine wave with linearly changing frequency (bandwidth) from both uniformly and nonuniformly distributed sample sets.

We have that, for $B(t)=t / 20000$ :

$$
f(t)=\sin [2 \pi \phi(t)]
$$

where $\phi(t)$ is the phase function, defined in terms of the instantaneous signal frequency (bandwidth) as follows:

$$
\phi(t)=\int_{0}^{t} B(\tau) d \tau=k_{1} t^{2}+k_{2} t
$$

For the bandwidth stipulated above we get that $k_{1}=1 / 40000$ and that $k_{2}=0$.

The uniform sampling sequence is $t_{n}=10 n$, and hence, at $t$ $=1000$ we are sampling at the Nyquist rate. In the

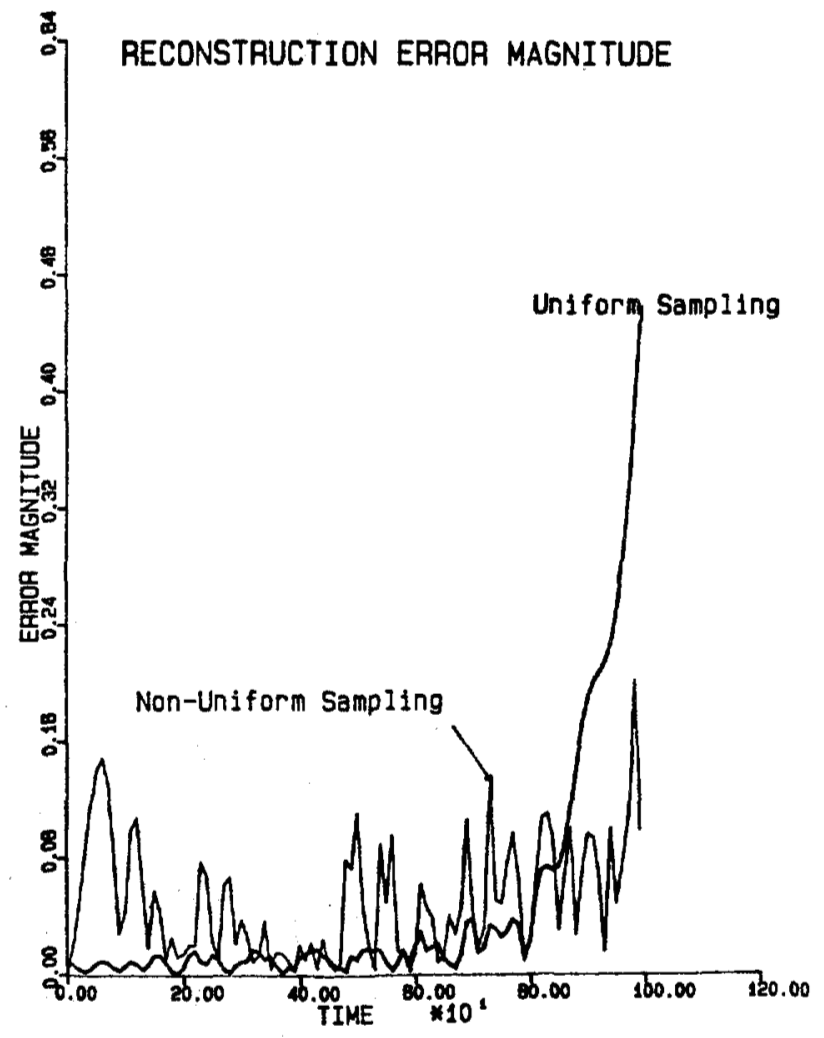

Fig. 9. Error magnitude of the reconstructed linear FM sine wave when sampled uniformly and nonuniformly.

nonuniform case, let us sample such that [from (12)]

$$
y(t)=C \int_{0}^{t}\left(2 \pi / \omega_{0}\right) B(\tau) d \tau=2 c \phi(t)
$$

The constant $c$ is chosen so that there are 100 samples in the interval [0, 1000]. We have, then, that

$$
\gamma(t)=c t^{2} / 20000
$$

We know that $\gamma\left(t_{n}\right)=n$. Therefore we must have that $\gamma(1000)=100$. This yields a value of 2 for $c$. We can now see that the sample sequence for the nonuniform case is, given by

$$
t_{n}=100 \sqrt{n}
$$

The function (36) was sampled at the uniform and nonuniform sample points, and then reconstructed using (1) and (12) truncated to 21 samples. The error magnitudes of the two reconstructions are shown in Fig. 9, along with the signal bandwidth. Notice that the error for the uniform case rises as the signal frequency rises because of the increasing aliasing and truncation errors, and that the error for the nonuniform case stays more or less constant, as expected. The total root mean square error for $10<t<990$ is 0.0982 for the uniform case and 0.0706 for the nonuniform case.

\section{Two Dimensions}

The following example illustrates the use of the twodimensional nonuniform sampling theory in the reconstruction of images from data obtained from aperture synthesis radio 


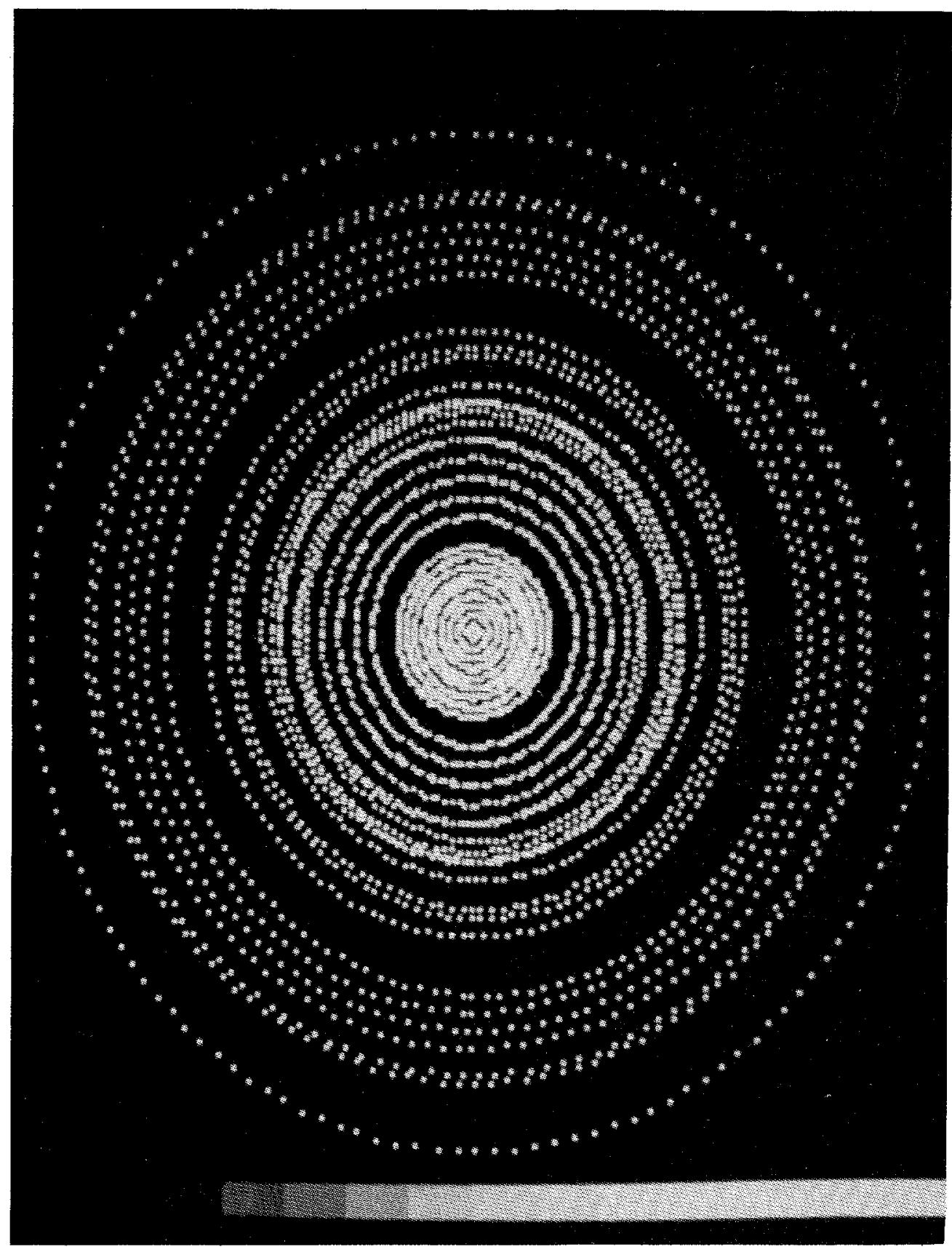

Fig. 10. The sample distribution in the spatial frequency plane for the proposed Canadian VLBI.

telescopes. The theory behind these astronomical imaging devices can be found in [9]. Basically, the output of an aperture synthesis radio telescope is a set of samples of the complex Fourier transform of the spatial radio intensity distribution in the small patch of sky that the telescope is viewing. These samples are not uniformly distributed, but are situated at equal angular intervals on sets of ellipses, as shown in Fig. 10.

To test the nonuniform reconstruction algorithm described in this paper on the VLBI (very long baseline interferometer) image reconstruction problem, we used the following procedure. Since there are no images available from the array at present, (the system is still in the planning stages) we use an optical image of a galaxy, in this case the Andromeda galaxy, shown in Fig. 11. We compute the Fourier transform of the galaxy image (the magnitude of which is shown in Fig. 12), and sample the real and imaginary parts of the transformed image at the sample points shown in Fig. 10. Note that, in practice, reliable measurements may be available for only the magnitude of the Fourier transform, and hence, the phase must be estimated from the magnitude, for example with the method of [7]. Since we are interested only in the function reconstruction, we will assume that both magnitude and phase of the Fourier transform are available. We then reconstruct the real and imaginary parts of the Fourier transform of the image from these samples using the algorithm described in this paper, and the method, described in [9], of Gaussian smearing. The Gaussian smearing technique involves the convolu- 


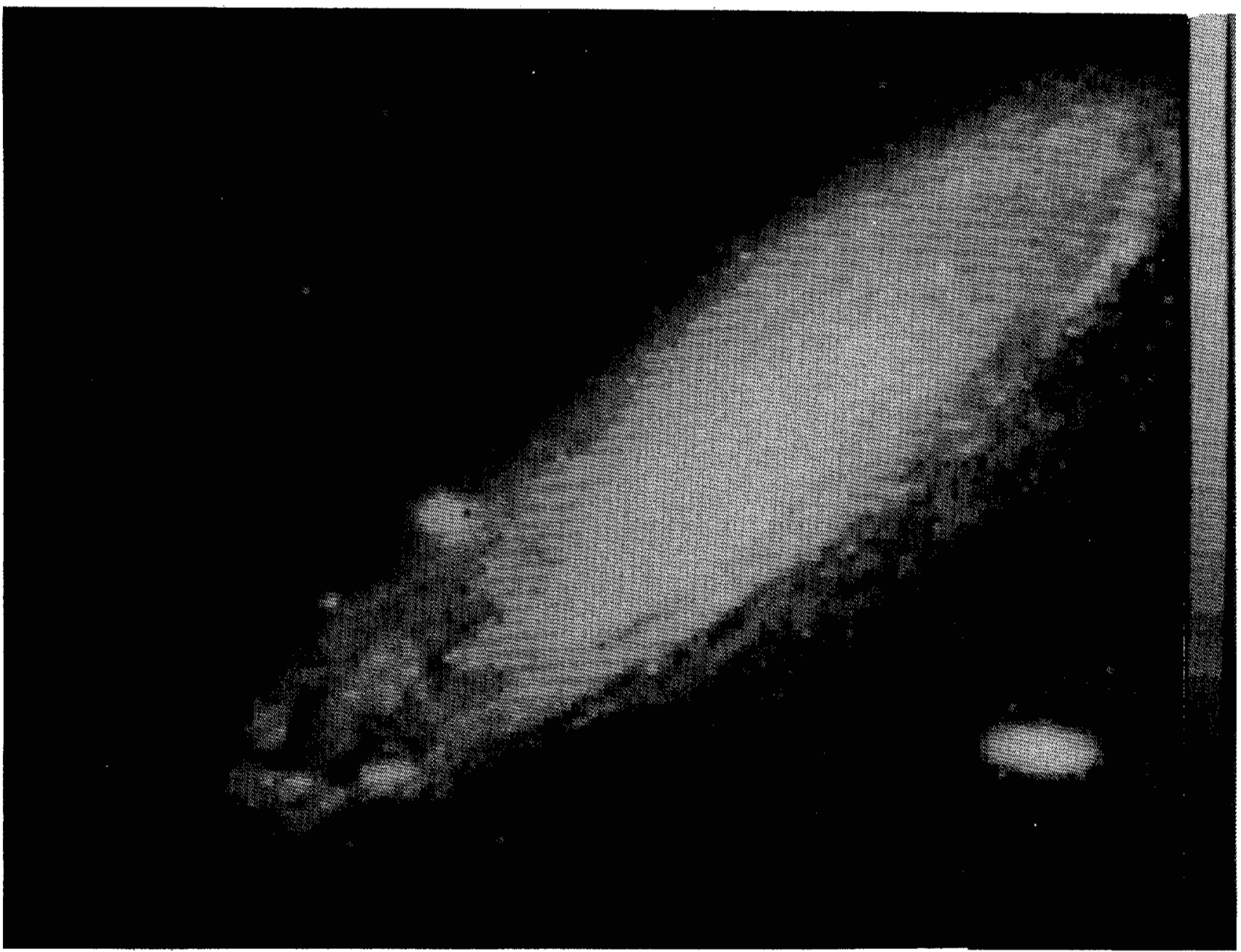

Fig. 11. The Andromeda galaxy visual image, used here as a test radio image.

tion of the sampled Fourier transform with a Gaussian function and resampling along a Cartesian lattice.

The reconstructed image resulting from each of these two methods is displayed in Figs. 13 and 14. The total square error for each reconstruction technique was measured. For the Gaussian smearing method the total square error was 215.2 and for the method of this paper the total square error was 158.3 (note that the total error was calculated over $128 \times 128$ points and the maximum function value was 255 ). It appears that the method described in this paper is superior to the Gaussian smearing method in terms of the minimization of square error (a nearest neighbor technique was also tested and performed far worse than either Gaussian smearing or the nonuniform reconstruction algorithm). The Gaussian smearing method was much faster than the nonuniform reconstruction algorithm, however. This may not be so important in applications, such as radio astronomy, where the data collection time is very long (on the order of hours for radio telescopes). Also, the bulk of the processing time for the algorithm described in this paper was taken up with computing $\bar{\gamma}$. However, for a fixed telescope 'geometry the sample positions $\left\{\bar{x}_{s}\right\}$ are also fixed so that $\bar{\gamma}$ need only be calculated once.
VIII. SUMMARY

We have presented a sampling theory which, under certain conditions, allows the exact reconstruction of $n$-dimensional functions from arbitrarily distributed samples. It has been shown that some nonband-limited functions, such as certain FM signals, can be reconstructed exactly with the methods of this paper. A heuristic algorithm is presented which implements the reconstruction process in two dimensions. Examples of the use of the nonuniform reconstruction methods are provided for the one- and two-dimensional case, showing the performance of these methods against other techniques of function sampling and reconstruction.

\section{APPENDIX}

\section{Proof of Theorems Stated in the Text}

Proof of Theorem 1: Proofs of this theorem can be found in many places. For a survey of these see [11].

Proof of Theorem 2: Since $h(\tau)$ is band limited to $\omega_{0}=\pi$, we can write (from Theorem 1):

$$
h(\tau)=\sum_{n=-\infty}^{\infty} h(n) g(\tau-n)
$$




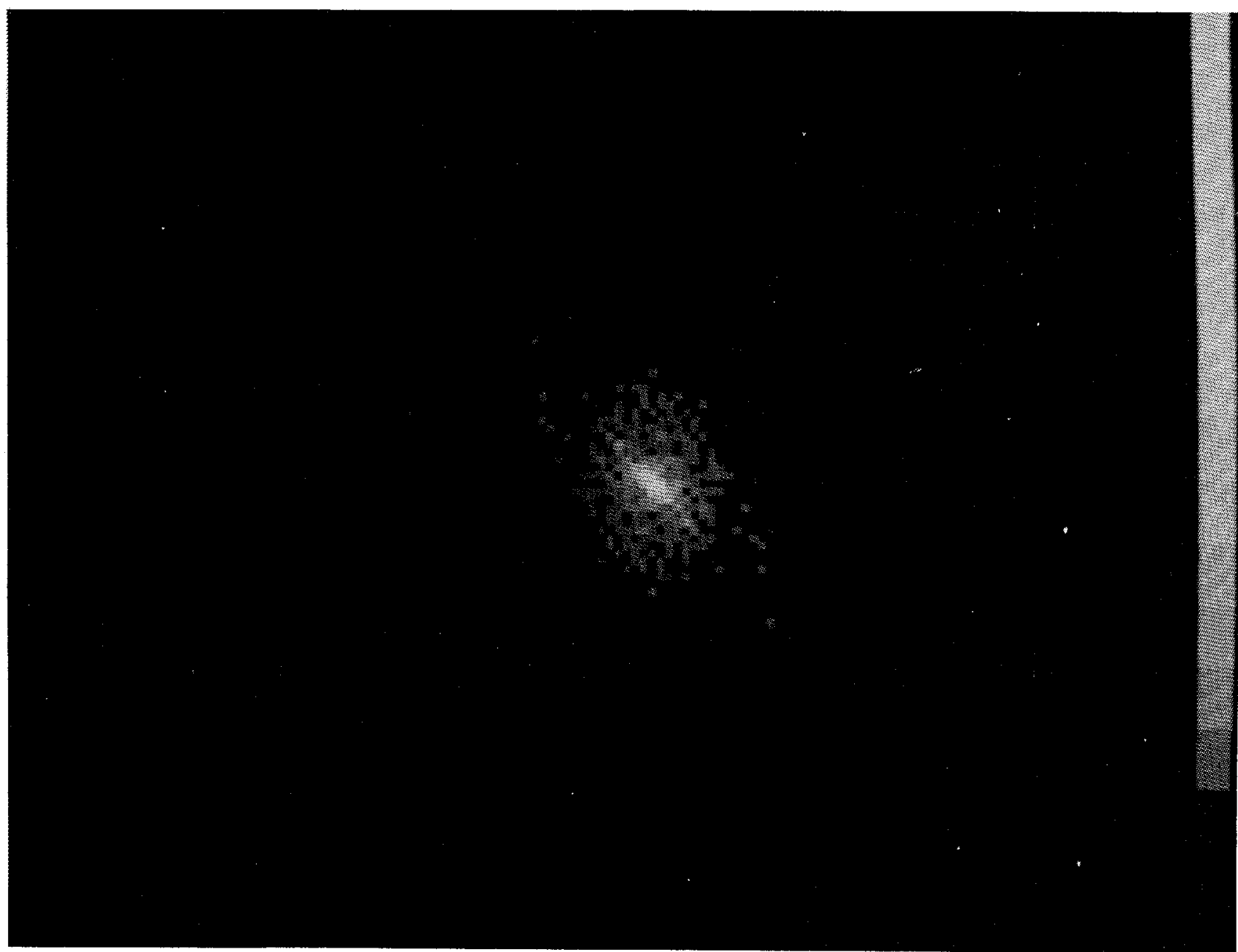

Fig. 12. The magnitude of the 2-D Fourier transform of Fig. 15.

Now, since a one-to-one continuous mapping $\gamma(t)$ exists such that $n=\gamma\left(t_{n}\right)$ and $\tau=\gamma(t)$, we have that

$$
h(\gamma(t))=\sum_{n=-\infty}^{\infty} h\left(\gamma\left(t_{n}\right)\right) g(\gamma(t)-n)
$$

Because $h(\gamma(t))=f(t)$ we have that

$$
f(t)=\sum_{n=-\infty}^{\infty} f\left(t_{n}\right) g(\gamma(t)-n)
$$

Q.E.D.

Proof of Theorem 3: For a proof of this theorem see [16]. Proof of Theorem 4: From Theorem 3 we have that

$$
h(\bar{\xi})=\sum_{\left\{\bar{\xi}_{s}\right\}} h\left(\bar{\xi}_{s}\right) g\left(\bar{\xi}-\bar{\xi}_{s}\right) .
$$

Now, since $\bar{\gamma}\left(\bar{x}_{s}\right)=\bar{\xi}_{s}$ and $\bar{\gamma}(\bar{x})=\bar{\xi}$, we have that

$$
h(\bar{\xi})=\sum_{\left\{\bar{x}_{s}\right\}} h\left(\bar{\gamma}\left(\bar{x}_{s}\right)\right) g\left(\bar{\gamma}(\bar{x})-\bar{\gamma}\left(\bar{x}_{s}\right)\right) .
$$

The condition that $h(\bar{\xi})=f(\bar{x})$, along with the condition that the Jacobian of the transformation $\bar{\gamma}$ be nonzero every- where, gives then that

$$
f(\bar{x})=\sum_{\left\{\bar{x}_{s}\right\}} f\left(\bar{x}_{s}\right) g\left(\bar{\gamma}(\bar{x})-\bar{\gamma}\left(\bar{x}_{s}\right)\right) .
$$

Proof of Theorem 5: A mapping $\bar{\gamma}(\bar{x})$ is one-to-one and continuous if the determinant of its Jacobian matrix $\mid \partial \bar{\gamma}(\bar{x}) /$ $\partial \bar{x} \mid$ is nonzero everywhere. At the interior points of $P_{x}$ (those points that are not vertex or link points of the partition), we have $\bar{\gamma}$ as defined in (27), (28), and (29). We can rewrite (27) as follows:

$$
\bar{\gamma}(\bar{x})=\Delta^{-1} \Gamma^{T}[(\bar{A}, \bar{B}) \bar{x}+\bar{C}]
$$

where

$$
\begin{gathered}
\Gamma=\left[\begin{array}{ll}
\Gamma_{1}^{1} & \Gamma_{1}^{2} \\
\Gamma_{2}^{1} & \Gamma_{2}^{2} \\
\Gamma_{3}^{1} & \Gamma_{3}^{2}
\end{array}\right] \\
\bar{A}=\left[\begin{array}{l}
\left(x_{2}^{2}-x_{3}^{2}\right) \\
\left(x_{3}^{2}-x_{1}^{2}\right) \\
\left(x_{1}^{2}-x_{2}^{2}\right)
\end{array}\right]
\end{gathered}
$$




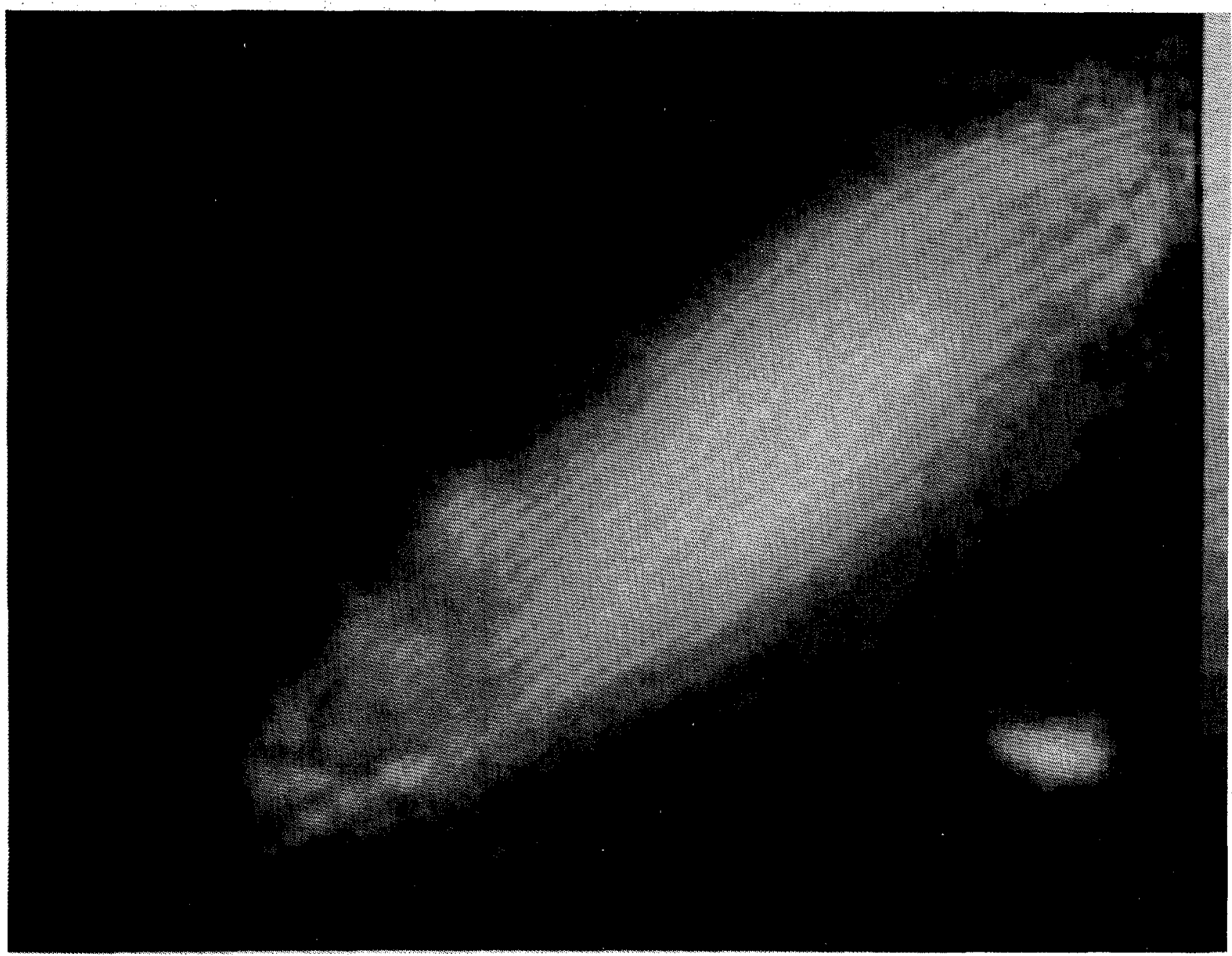

Fig. 13. The reconstructed image using the nonuniform reconstruction method described in this paper.

$$
\bar{B}=\left[\begin{array}{c}
\left(x_{3}^{1}-x_{2}^{1}\right) \\
\left(x_{1}^{1}-x_{3}^{1}\right) \\
\left(x_{2}^{1}-x_{1}^{1}\right)
\end{array}\right] .
$$

The vector $\bar{C}$ is of no consequence in this proof as it does not appear in the expression for the Jacobian. $\Delta$ is as given in (29). After some algebraic manipulation we get that

$$
J=\Delta^{-2}\left|\Gamma^{T}(\bar{A}, \bar{B})\right|=\alpha \beta / \Delta^{2}
$$

where

$$
\begin{gathered}
\alpha=x_{1}^{2}\left(x_{3}^{1}-x_{2}^{1}\right)+x_{2}^{2}\left(x_{1}^{1}-x_{3}^{1}\right)+x_{3}^{2}\left(x_{2}^{1}-x_{1}^{1}\right) \\
\beta=\Gamma_{1}^{2}\left(\Gamma_{3}^{1}-\Gamma_{2}^{1}\right)+\Gamma_{2}^{2}\left(\Gamma_{1}^{1}-\Gamma_{3}^{1}\right)+\Gamma_{3}^{2}\left(\Gamma_{2}^{1}-\Gamma_{1}^{1}\right) .
\end{gathered}
$$

It can be shown that $\alpha=0$ iff the points $\bar{x}_{1}, \bar{x}_{2}, \bar{x}_{3}$ are collinear, and that $\beta=0$ iff the points $\Gamma_{1}, \Gamma_{2}, \Gamma_{3}$ are collinear. Since the points $\Gamma_{i}$ are not collinear, $\beta \neq 0$ and $\bar{\gamma}$ will have a nonzero Jacobian at the interior points of $P_{x}$ when the points in the set $V(\bar{x})$ are not collinear.

In order that $\bar{\gamma}$ be one-to-one and continuous at the links of $P_{x}$ we must ensure that the value of the Jacobians on either side of an link of $P_{x}$ have the same sign.

Consider Figs. 15 and 16, which illustrate the mapping of points in $\bar{x}$ space to the hexagonal lattice in $\bar{\xi}$ space. We have mapped the points $\bar{x}_{1}, \bar{x}_{2}, \bar{x}_{3}$ in $\bar{x}$ space into the points $\Gamma_{1}, \Gamma_{2}$, $\Gamma_{3}$ in $\bar{\xi}$ space, creating the partition regions $P_{1}$ and $D_{1}$.

Let us assume that $\alpha>0$ and that $\beta>0$ for this mapping. We now wish to map a sample point in $\bar{x}$ space to the point $\Gamma_{4}$ of the hexagonal lattice in $\bar{\xi}$ space, to create the partiton regions $P_{2}, D_{2}$ that share a common link with $P_{1}$ and $D_{1}$. Imagine that $\Gamma_{4}$ was not constrained to lie on a vertex of the hexagonal sampling lattice, but could lie anywhere in $\bar{\xi}$ space. It can be seen that if $\Gamma_{4}$ was to lie anywhere on the line through $\Gamma_{1}$ and $\Gamma_{2}$ that $\beta$ would be zero (as the points $\Gamma_{1}, \Gamma_{2}$ and $\Gamma_{4}$ are collinear). Furthermore, it can be seen that only along this line can $\beta$ be zero. Hence if $\Gamma_{4}$ lies on the same side of the line through $\Gamma_{1}$ and $\Gamma_{2}$ as does $\Gamma_{3}$ then $\beta$ is positive, and if it lies on the opposite side then $\beta$ is negative. Now since we have the constraint that $\Gamma_{4}$ must lie on the hexagonal sampling lattice, it can be seen that $\beta$ for the region formed by $\Gamma_{1}, \Gamma_{2}$ and $\Gamma_{4}$ is negative. Now for the Jacobian of $\bar{\gamma}$ to have the same sign in $P_{2}$ as in $P_{1}, \alpha$ for $P_{2}$ must also be negative. Hence $\bar{x}_{4}$ must lie on the side of the line through $\bar{x}_{1}$ and $\bar{x}_{2}$ opposite to $\bar{x}_{3}$. In other words the region formed by points $x_{1}, x_{2}$, and $x_{3}$ must not overlap the region formed by points $x_{1}, x_{2}$, and $x_{4}$. That is, $P_{x}$ must be a tessellation for the mapping function $\bar{\gamma}$ to be one-to-one and continuous.

Q.E.D. 


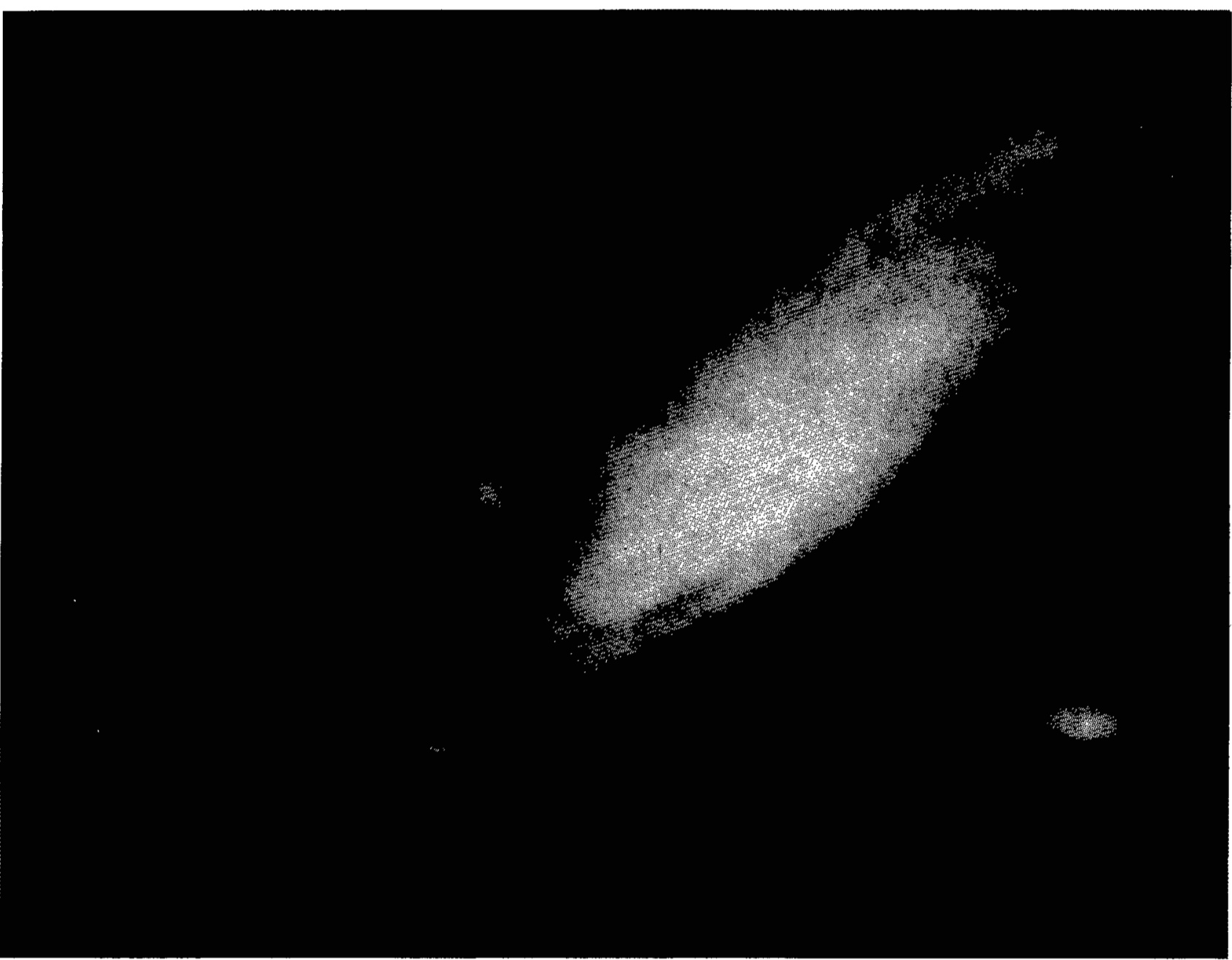

Fig. 14. The reconstructed image using the Gaussian smearing method.

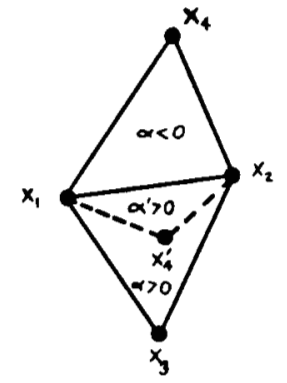

Fi :. 15. A portion of the partition $P_{X 0}$ in $\bar{x}$ space.

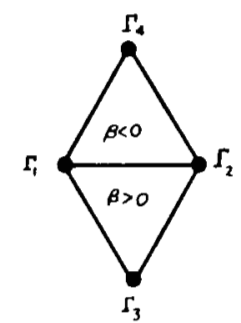

Fig. 16. A portion of the partition $D_{h 0}$ in $\bar{\xi}$ space.

\section{REFERENCES}

[1] M. Abramowitz and I. A. Stegun, Handbook of Mathematical Functions. New York: Dover, 1965.

[2] N. Ahuja and B. Schacter, Pattern Models. New York: Wiley, 1983.

[3] F. J. Beutler, "Error free recovery of signals from irregularly spaced samples," SIAM Rev., vol. 8, no. 3, pp. 328-335, 1966.

[4] J. J. Clark, "Modification of Hall's efficient spiral search to allow metrically ordered searches," to be published.

[5] W. E. L. Grimson, "A computational theory of visual surface interpolation," Phil. Trans. R. Soc. Lond. B, vol. 298, pp. 395-427, 1982.

[6] R. W. Hall, "Efficient spiral search in bounded spaces," IEEE Trans. Pattern Anal. Mach. Intell., vol. PAMI-4, pp. 208-214, 1982.

[7] M. H. Hayes, "The reconstruction of a multidimensional sequence from the phase or the magnitude of its Fourier transform," IEEE Trans. Acoust., Speech, Signal Processing, vol. ASSP-30, pp. 140154, 1983.

[8] J. R. Higgins, "A sampling theorem for irregularly spaced sample points," IEEE Trans. Inform. Theory, Sept. 1976.

[9] R. W. Hjellming, "An introduction to the radio astronomy very large array," National Radio Astronomy Observatory, United States of America, 1982.

[10] K. Horiuchi, "Sampling principle for continuous signals with timevarying bands," Inform. Control, vol. 13, pp. 53-61, 1968.

[11] A. J. Jerri, "The Shannon sampling theorem-Its various extensions and applications: A tutorial review," Proc. IEEE, vol. 65, pp. 1565-1596, Nov. 1977.

[12] R. M. Mersereau, "The processing of hexagonally sampled twodimensional signals," Proc. IEEE, vol. 67, pp. 930-949, 1979.

[13] R. M. Mersereau and T. C. Speake, "The processing of periodically 
sampled multidimensional signals,"' IEEE Trans. Acoust., Speech, Signal Processing, vol. 31, pp. 188-194, Feb. 1983.

[14] S. X. Pan and A. C. Kak, "A computational study of reconstruction algorithms for diffraction tomography: Interpolation versus filtered Backpropogation," IEEE Trans. Acoust., Speech, Signal Processing, vol. ASSP-31, pp. 1262-1275, Oct. 1983.

[15] A. Papoulis, "Error analysis in sampling theory," Proc. IEEE, vol. 54, pp. 947-955, July 1966.

[16] D. P. Petersen and D. Middleton, "Sampling and reconstruction of wave-number limited functions in $N$-dimensional Euclidean spaces," Inform. Control, vol. 5, pp. 279-323, 1962.

[17] D. Terzopoulos, "Multilevel reconstruction of visual surfaces: Variational principles and finite element methods," MIT, Cambridge, MA, Al memo 671, 1982.

[18] K. Yao and J. B. Thomas, "On some stability and interpolating properties of non-uniform sampling expansions," IEEE Trans. Circuit Theory, vol. CT-14, pp. 404-408, 1967.

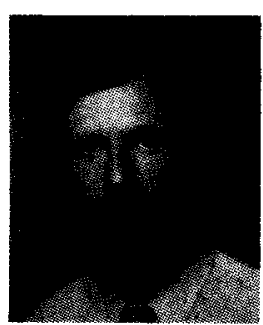

James J. Clark was born in Vancouver, B.C. in 1957. He received the B.A.Sc. degree in electrical engineering from the University of British Columbia, Vancouver, B.C., Canada, in 1980, and completed his Ph.D. studies in the same department in 1985.

He has done research at the National Research Council of Canada, and has consulted for the Faculty of Forestry at the University of British Columbia. He is currently with the Division of Applied Sciences, Harvard University, Cambridge, MA. His interests include computational vision, image processing, robotics, and multidimensional signal processing.

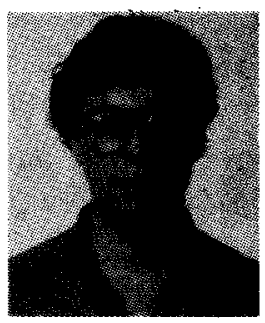

Matthew R. Palmer was born in Montreal, Quebec, Canada, on April 3, 1958. He received the B.A.Sc. degree in electrical engineering from the University of British Columbia in 1981. He completed the M.A.Sc. degree requirements in the Department of Electrical Engineering and the Imaging Research Centre at U.B.C. in 1985.

From 1981 to 1983 he was a Research Assistant, and a member of the technical staff with the Department of Electrical Engineering, University of British Columbia, Vancouver, B.C., Canada. He is currently with the TRIUMF/UBC PETT project. His research interests are in the areas of image processing and medical imaging.

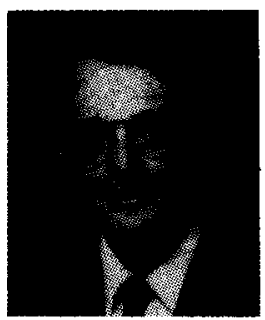

Peter D. Lawrence (S'64-M'73) received the B.A.Sc. degree from the University of Toronto, Toronto, Ont., Canada, in 1965, the M.Sc. degree from the University of Saskatchewan, Saskatoon, Sask., Canada, in 1967, and the Ph.D. degree from Case Western Reserve University, Cleveland, $\mathrm{OH}$, in 1970 .

From 1970 to 1972 , he was a Guest Researcher with the Department of Applied Electronics, Chalmers University of Technology, Goteborg, Sweden. From 1972 to 1974 , he was a Lecturer and Research Staff Member at Massachusetts Institute of Technology, Cambridge. $\mathrm{He}$ is presently a Full Professor with the Department of Electrical Engineering, University of British Columbia, Vancouver, B.C., Canada. His research interests are in computer applications in biomedical signal analysis and robotics. 